\title{
Role of Low-Molecular-Mass Penicillin-Binding Proteins, NagZ and AmpR in AmpC $\beta$-lactamase Regulation of Yersinia enterocolitica
}

\section{OPEN ACCESS}

Edited by:

Dongsheng Zhou,

Beijing Institute of Microbiology and

Epidemiology, China

Reviewed by:

Fang Huang,

Beijing Center for Disease Prevention

and Control, China

L. F. Wu,

Centre National de la Recherche

Scientifique (CNRS), France

*Correspondence:

Xin Wang

wangxin@icdc.cn

Shihe Shao

shaoshihe2006@163.com

${ }^{\dagger}$ These authors have contributed equally to this work

Received: 20 July 2017 Accepted: 14 September 2017 Published: 27 September 2017

Citation:

Liu C, Li C, Chen Y, Hao H, Liang J,

Duan R, Guo Z, Zhang J, Zhao Z, Jing $H$, Wang $X$ and Shao $S$ (2017)

Role of Low-Molecular-Mass

Penicillin-Binding Proteins, NagZ and

AmpR in AmpC $\beta$-lactamase

Regulation of Yersinia enterocolitica.

Front. Cell. Infect. Microbiol. 7:425.

doi: 10.3389/fcimb.2017.00425

\begin{abstract}
Chang Liu ${ }^{1,2+}$, Chuchu $\mathrm{Li}^{1,2+}$, Yuhuang Chen ${ }^{2}$, Huijing Hao ${ }^{2}$, Junrong Liang ${ }^{2}$, Ran Duan ${ }^{2}$, Zhaoke Guo ${ }^{2}$, Jing Zhang ${ }^{2}$, Zhongzhi Zhao ${ }^{3}$, Huaiqi Jing ${ }^{2}$, Xin Wang ${ }^{2 \star}$ and Shihe Shao ${ }^{1 *}$

${ }^{1}$ Department of Pathogenic Biology, School of Medical Science, Jiangsu University, Zhenjiang, China, ${ }^{2}$ National Institute for Communicable Disease Control and Prevention, Chinese Center for Disease Control and Prevention, State Key Laboratory for Infectious Disease Prevention and Control, Collaborative Innovation Center for Diagnosis and Treatment of Infectious Diseases, Beijing, China, ${ }^{3}$ Qinghai Institute for Endemic Diseases Prevention and Control, Xining, China
\end{abstract}

Yersinia enterocolitica encodes a chromosomal AmpC $\beta$-lactamase under the regulation of the classical ampR-ampC system. To obtain a further understanding to the role of low-molecular-mass penicillin-binding proteins (LMM PBPs) including PBP4, PBP5, PBP6, and PBP7, as well as NagZ and AmpR in ampC regulation of $Y$. enterocolitica, series of single/multiple mutant strains were systematically constructed and the ampC expression levels were determined by luxCDABE reporter system, reverse transcription-PCR (RT-PCR) and $\beta$-lactamase activity test. Sequential deletion of PBP5 and other LMM PBPs result in a continuously growing of ampC expression level, the $\beta$-lactamse activity of quadruple deletion strain YE $\Delta 4 \Delta 5 \Delta 6 \Delta 7$ (pbp4, pbp5, pbp6, and $p b p 7$ inactivated) is approached to the YE $\Delta \mathrm{D} 123$ (ampD1, ampD2, and ampD3 inactivated). Deletion of nagZ gene caused two completely different results in YE $\Delta D 123$ and $\mathrm{YE} \Delta 4 \Delta 5 \Delta 6 \Delta 7, \mathrm{NagZ}$ is indispensable for $\mathrm{YE} \Delta 4 \Delta 5 \Delta 6 \Delta 7$ ampC derepression phenotype but dispensable for $\mathrm{YE} \Delta \mathrm{D} 123$. AmpR is essential for ampC hyperproduction in these two types of strains, inactivation of $A m p R$ notable reduced the ampC expression level in both YE $\Delta \mathrm{D} 123$ and $\mathrm{YE} \Delta 4 \Delta 5 \Delta 6 \Delta 7$.

Keywords: Yersinia enteocolitica, AmpC $\beta$-lactamase, AmpD, PBPs, NagZ, AmpR

\section{INTRODUCTION}

Yersinia enterocolitica, a member of Enterobacteriaceae, is a zoonotic pathogen widely distributed in nature (Wang et al., 2011; Liang et al., 2012). Most Y. enterocolitica exhibits intrinsic resistance to $\beta$-lactm antibiotics by the production of chromosomally encoded $\beta$-lactamases called BlaA (a class A enzyme showing constitutive expression) and BlaB (an inducible AmpC-type $\beta$-lactamase), respectively (Cornelis and Abraham, 1975; Bent and Young, 2010).

The process of ampC (blaB) regulation is tightly linked to the peptidoglycan recycling and controlled by AmpG, AmpD, AmpR, and NagZ (Vollmer et al., 2008; Zeng and Lin, 2013). Briefly, peptidoglycan degradation products including GlcNAc-1,6-anhydromuropeptide is transported into the cytoplasm by AmpG and further hydrolyzedcosaminidase) to yielding 1,6-anhydromuropeptides, which is the AmpR activator ligand for ampC derepression (Zamorano et al., 2010; Huang et al., 2012; Yang et al., 2014). On the other hand, 
the stem peptides of GlcNAc-1,6-anhydromuropeptide and 1,6-anhydromuropeptides can be removed by AmpD (N-acetylmuramyl-L-alanine amidase) and eventually recycled into UDP-MurNAc-pentapeptide, which is the AmpR repressor ligand to repress ampC expression level (Juan et al., 2006; Balasubramanian et al., 2015; Liu et al., 2016). Penicillin-binding proteins (PBPs) also play an important role in ampC regulation (Sanders et al., 1997; Pfeifle et al., 2000). Recent studies have found that in P. aeruginosa, PBP4 (DacB), PBP5 (DacC), and $\mathrm{PBP} 7(\mathrm{PbpG})$ are involved in $a m p C$ regulation, and $\mathrm{PBP} 4$ is the major cause of $a m p C$ derepressed in clinical strains (Moya et al., 2009; Ropy et al., 2015).

Theoretically, NagZ is indispensable in chromosomal ampC derepression. In $P$. aeruginosa, nag $Z$ inactivation dramatically reduces the $\beta$-lactam resistance of both $\mathrm{PAO} \triangle \mathrm{ampD}(a m p D$ inactivation) and $\mathrm{PAO} \Delta d a c B$ ( $p b p 4$ inactivation; Zamorano et al., 2010). However, although nagZ inactivation nearly abolished the basal-level derepressed $\beta$-lactamase activity of $\mathrm{KJ} \Delta \mathrm{ampDI}$ ( $a m p D$ inactivation), it did not affect the $\beta$-lactamase activity of $\mathrm{KJ} \Delta \mathrm{mrcA}$ ( $p b p 1 a$ inactivation) in Stenotrophomonas maltophilia (Huang et al., 2012).

Since the effects of the above-mentioned genes in $Y$. enterocolitica were seldom reported, we elucidated the role of low-molecular-mass penicillin-binding proteins (LMM PBPs) (PBP4, PBP5, PBP6, and PBP7), NagZ and AmpR in the $Y$. enterocolitica ampC regulation. Firstly, we investigated the effects of each LMM PBP on the expression of AmpC $\beta$-lactamase by monitoring the $a m p C$ promoter activity from a series of LMM PBPs mutant strains and confirmed by quantitative reverse transcription-PCR (qRT-PCR). Secondly, nagZ gene was deleted in two $\operatorname{ampC}$ derepressed strains YE $\Delta \mathrm{D} 123$ and YE $\Delta 4 \Delta 6 \Delta 5 \Delta 7$ to determine the role for $a m p C$ expression.

\section{MATERIALS AND METHODS}

\section{Bacterial Strains, Plasmids, Primers, and Growth Conditions}

Strains and plasmids used in this study were listed in Table $\mathbf{1 .}$ Individual genes were deleted initially from $Y$. enterocolitica subsp. palearctica 105.5R(r) (Wang et al., 2011). Luria-Bertani (LB) agar plates and broth were used as culture media for $Y$. enterocolitica $\left(28^{\circ} \mathrm{C}\right)$ and Escherichia coli $\left(37^{\circ} \mathrm{C}\right)$. For induction assay, cefoxitin was used according to the references (Guerin et al., 2015; Liu et al., 2016).

\section{Construction of $Y$. enterocolitica Mutant Strains}

Knockout mutant strains were constructed using the method described previously (Chen et al., 2015; Liang et al., 2016; Liu et al., 2016). Briefly, the deletion mutants were constructed by double-crossover homologous recombination between wildtype strain chromosome and plasmids $\mathrm{p} \Delta \mathrm{NagZ}, \mathrm{p} \Delta \mathrm{AmpR}$, $\mathrm{p} \triangle \mathrm{PBP} 4, \mathrm{p} \triangle \mathrm{PBP} 5, \mathrm{p} \triangle \mathrm{PBP} 61$, and $\mathrm{p} \triangle \mathrm{PBP} 7$. To evaluate the role of PBP4 (WP_005175403.1), PBP5 (WP_005158391.1) PBP6 (WP_023160783.1), and PBP7 (WP_005158897.1) in $Y$. enterocolitica $105.5 \mathrm{R}(\mathrm{r})$ ampC regulation, we constructed four
TABLE 1 | Strains and plasmids used in this study.

\begin{tabular}{|c|c|c|}
\hline $\begin{array}{l}\text { Strains or } \\
\text { plasmid }\end{array}$ & $\begin{array}{l}\text { Genotype or relevant } \\
\text { characteristics }\end{array}$ & $\begin{array}{l}\text { Source or } \\
\text { references }\end{array}$ \\
\hline \multicolumn{3}{|c|}{ Yersinia enterocolitica } \\
\hline $105.5 R(r)$ & Wild type; completely sequenced & Wang et al., 2011 \\
\hline YE $\Delta Z$ & 105.5R(r) nagZ deletion mutant & This work \\
\hline YEAD123 & $\begin{array}{l}105.5 \mathrm{R}(\mathrm{r}) \mathrm{ampD} 1, \mathrm{ampD2}, \mathrm{ampD} 3 \\
\text { triple mutant }\end{array}$ & Liu et al., 2016 \\
\hline YE $\Delta D 123 \Delta Z$ & $\begin{array}{l}\text { 105.5R(r) ampD1, ampD2, ampD3, } \\
\text { nagZ quadruple mutant }\end{array}$ & This work \\
\hline $\mathrm{YE} \Delta \mathrm{D} 123 \Delta \mathrm{R}$ & $\begin{array}{l}\text { 105.5R(r) ampD1, ampD2, } a m p D 3, \\
\text { ampR quadruple mutant }\end{array}$ & This work \\
\hline YE $\Delta 4$ & 105.5R(r) pbp4 deletion mutant & This work \\
\hline YE $\Delta 5$ & 105.5R(r) pbp5 deletion mutant & This work \\
\hline YE $\Delta 6$ & 105.5R(r) pbp6 deletion mutant & This work \\
\hline YE $\Delta 7$ & 105.5R(r) pbp7 deletion mutant & This work \\
\hline $\mathrm{YE} \Delta 4 \Delta 5$ & 105.5R(r) pbp4, pbp5 double mutant & This work \\
\hline YE $\Delta 4 \Delta 6$ & 105.5R(r) pbp4, pbp6 double mutant & This work \\
\hline YE $\Delta 4 \Delta 7$ & 105.5R(r) pbp4, pbp7 double mutant & This work \\
\hline YE $\Delta 5 \Delta 6$ & 105.5R(r) pbp5, pbp6 double mutant & This work \\
\hline YE $\Delta 5 \Delta 7$ & 105.5R(r) pbp5, pbp7 double mutant & This work \\
\hline YE $\Delta 6 \Delta 7$ & 105.5R(r) pbp6, pbp7 double mutant & This work \\
\hline YE $\Delta 4 \Delta 5 \Delta 6$ & $\begin{array}{l}105.5 \mathrm{R}(\mathrm{r}) \mathrm{pbp} 4, \mathrm{pbp} 5, \mathrm{pbp} 6 \text { triple } \\
\text { mutant }\end{array}$ & This work \\
\hline YE $\Delta 4 \Delta 5 \Delta 7$ & $\begin{array}{l}105.5 \mathrm{R}(\mathrm{r}) \mathrm{pbp} 4, \mathrm{pbp} 5, \mathrm{pbp} 7 \text { triple } \\
\text { mutant }\end{array}$ & This work \\
\hline YE $\Delta 4 \Delta 6 \Delta 7$ & $\begin{array}{l}105.5 \mathrm{R}(\mathrm{r}) \mathrm{pbp} 4, \mathrm{pbp} 6, \mathrm{pbp} 7 \text { triple } \\
\text { mutant }\end{array}$ & This work \\
\hline YE $\Delta 5 \Delta 6 \Delta 7$ & $\begin{array}{l}105.5 \mathrm{R}(\mathrm{r}) \mathrm{pbp} 5, \mathrm{pbp} 6, \mathrm{pbp} 7 \text { triple } \\
\text { mutant }\end{array}$ & This work \\
\hline YE $\Delta 4 \Delta 5 \Delta 6 \Delta 7$ & $\begin{array}{l}\text { 105.5R(r) pbp4, pbp5, pbp6, pbp7, } \\
\text { quadruple mutant }\end{array}$ & This work \\
\hline YE $\Delta 4 \Delta 5 \Delta 6 \Delta 7 \Delta Z$ & $\begin{array}{l}105.5 \mathrm{R}(\mathrm{r}) \mathrm{pbp} 4, \mathrm{pbp} 5, \mathrm{pbp} 6, \mathrm{pbp} 7 \\
\text { nagZ quintuple mutant }\end{array}$ & This work \\
\hline YE $\Delta 4 \Delta 5 \Delta 6 \Delta 7 \Delta \mathrm{R}$ & $\begin{array}{l}\text { 105.5R(r) pbp4, pbp5, pbp6, pbp7, } \\
\text { ampR quintuple mutant }\end{array}$ & This work \\
\hline \multicolumn{3}{|l|}{ E. coli } \\
\hline S17 $\lambda$ pir & $\begin{array}{l}\lambda \text {-pir R6K(thi thr leu ton lacY supE } \\
\text { recA::RP4-2Tc::Mu) }\end{array}$ & Simon et al., 1983 \\
\hline \multicolumn{3}{|l|}{ PLASMIDS } \\
\hline pDS132 & $\begin{array}{l}\text { CmR; Conditionally replicating vector; } \\
\text { R6K origin, mobRK } 4 \text { transfer origin, } \\
\text { sucrose-inducible sacB }\end{array}$ & $\begin{array}{l}\text { Philippe et al., } \\
2004\end{array}$ \\
\hline $\mathrm{p} \Delta \mathrm{NagZ}$ & $\begin{array}{l}\text { CmR; pDS132 containing } 5^{\prime} \text { and } 3^{\prime} \\
\text { flanking sequence of nag } Z\end{array}$ & This work \\
\hline $\mathrm{p} \triangle \mathrm{PBP} 4$ & $\begin{array}{l}\text { CmR; pDS132 containing } 5^{\prime} \text { and } 3^{\prime} \\
\text { flanking sequence of } p b p 4\end{array}$ & This work \\
\hline $\mathrm{p} \triangle \mathrm{PBP} 5$ & $\begin{array}{l}\text { CmR; pDS132 containing } 5^{\prime} \text { and } 3^{\prime} \\
\text { flanking sequence of } p b p 5\end{array}$ & This work \\
\hline $\mathrm{p} \triangle \mathrm{PBP} 6$ & $\begin{array}{l}\text { CmR; pDS132 containing } 5^{\prime} \text { and } 3^{\prime} \\
\text { flanking sequence of } p b p 6\end{array}$ & This work \\
\hline $\mathrm{p} \triangle \mathrm{PBP} 7$ & $\begin{array}{l}\text { CmR; pDS132 containing } 5^{\prime} \text { and } 3^{\prime} \\
\text { flanking sequence of } p b p 7\end{array}$ & This work \\
\hline $\mathrm{p} \Delta \mathrm{AmpR}$ & $\begin{array}{l}\text { CmR; pDS132 containing } 5^{\prime} \text { and } 3^{\prime} \\
\text { flanking sequence of } a m p R\end{array}$ & This work \\
\hline pLUXampC & $\begin{array}{l}\text { CmR; pBBRlux containing promoter } \\
\text { sequence of } a m p C\end{array}$ & Liu et al., 2016 \\
\hline pNagZ & $\begin{array}{l}\text { TcR; pSRKTc containing 105.5R(r) } \\
\text { nagZ gene }\end{array}$ & This work \\
\hline
\end{tabular}


single mutant strains: $\mathrm{YE} \Delta 4$ ( $p b p 4$ inactivation), YE $\Delta 5$ ( $p b p 5$ inactivation), YE $\Delta 6$ ( $p b p 6$ inactivation), and $\mathrm{YE} \Delta 7$ ( $p b p 7$ inactivation); six double mutant strains: $\mathrm{YE} \Delta 4 \Delta 5$, YE $\Delta 4 \Delta 6$, $\mathrm{YE} \Delta 4 \Delta 7$, YE $\Delta 5 \Delta 6$, YE $\Delta 5 \Delta 7$, and $\mathrm{YE} \Delta 6 \Delta 7$; four triple mutant strains: YE $\Delta 4 \Delta 5 \Delta 6$, YE $\Delta 4 \Delta 5 \Delta 7$, YE $\Delta 4 \Delta 6 \Delta 7$, and YE $\Delta 5 \Delta 6 \Delta 7$; and one quadruple mutant strain: YE $\Delta 4 \Delta 5 \Delta 6 \Delta 7$ (Table 1). The deletion mutants were identified by colony PCR firstly and then sequenced to confirm the in-frame deletion. Multiple deletion strains were sequentially constructed from the single mutant by use of the same procedure.

\section{Measurement of the ampC Promoter Activity}

The method of measuring the $a m p C$ promoter activity with the luxCDABE reporter system was reported previously (Liu et al., 2016). The reporter plasmid pLUXampC was transferred into the tested strains, and the luminescence was measured by using an Infinite M200 Pro spectrophotometer. The value of luminescence/OD600 was used to assess the ampC promoter activity.

\section{Determination of $\beta$-Lactamase Activity and Antibiotic Susceptibility Testing}

Specific $\beta$-lactamase activities were spectrophotometrically determined with nitrocefin (Oxoid) as a substrate as previously described (Liu et al., 2016). One unit of $\beta$-lactamase activity $(\mathrm{U} / \mathrm{mg})$ was defined as the number of nanomoles of nitrocefin hydrolyzed per minute per milligram of protein. Antibiotic susceptibility was determined using the standard 2-fold serial broth microdilution method according to the Guidelines of the Clinical Laboratory Standards Institute (CLSI, 2015).

\section{N-Acetyl- $\beta$-Glucosaminidase Activity Assay}

The N-acetyl-glucosaminidase activity of the whole cell lysates of wild-type strain $105.5 \mathrm{R}(\mathrm{r})$ and $\mathrm{YE} \Delta \mathrm{Z}$ were measured using 4-nitrophenyl $\mathrm{N}$-acetyl- $\beta$-D-glucosaminide as a chromogenic substrate (Sigma). The presence of $\mathrm{p}$-nitrophenol were detected by monitoring the optical density at $405 \mathrm{~nm}$ by $10 \mathrm{~h}$ continuously.

\section{Complementation Assay}

The ORF of nag $Z$ was amplified and cloned into the broad-hostrange expression vector pSRKTc to construct plasmid pNagZ. Transformants were selected on $10 \mu \mathrm{g} / \mathrm{ml}$ tetracycline Yersinia selective LB plates, acquisition of the appropriate plasmid was confirmed by colony PCR.

\section{RESULTS}

\section{Role of LMM PBPs in the Expression of AmpC $\beta$-Lactamase}

After a series of LMM PBPs mutant strains were constructed, reporter plasmid pLUXampC was used to monitor the $a m p C$ expression level (Liu et al., 2016). As shown in Figure 1, deletion $p b p 5$ caused a visible increase in the ampC promoter activity under both basal and induced conditions; but deletion of $p b p 4, p b p 6$, and $p b p 7$ did not affect the AmpC expression obviously. In the group of double and triple mutant strains,
ampC derepression only appeared in $\Delta p b p 5$ background, the $a m p C$ promoter activity of $\mathrm{YE} \Delta 4 \Delta 5$, YE $\Delta 5 \Delta 6$, and $\mathrm{YE} \Delta 5 \Delta 7$ exhibited a marked rise compared with YE $\Delta 4 \Delta 6$, YE $\Delta 4 \Delta 7$, or YE $\Delta 6 \Delta 7$. The level of ampC expression keep increasing in triple mutant strains YE $\Delta 4 \Delta 5 \Delta 6$, YE $\Delta 4 \Delta 5 \Delta 7$, and YE $\Delta 5 \Delta 6 \Delta 7$, but not in YE $\Delta 4 \Delta 6 \Delta 7$. Finally, the quadruple deletion strain YE $\Delta 4 \Delta 5 \Delta 6 \Delta 7$ displayed the highest level of $a m p C$ promoter activity. These results suggested that PBP5 plays the most important roles in $Y$. enterocolitica $\operatorname{ampC}$ regulation. The qRT-PCR assay reconfirmed the results observed from $a m p C$ promoter activity assay (Table 2 ).

\section{Role of NagZ in AmpC Derepression of Y. enterocolitica}

In agreement with our previous data (Liu et al., 2016), AmpD deletion strain YE $\triangle \mathrm{D} 123$ exhibit a derepression phenotype, and the $\beta$-lactamase activity of YE $\Delta \mathrm{D} 123$ is slightly higher than YE $\Delta 4 \Delta 5 \Delta 6 \Delta 7$ (Figure 2). To evaluate the role of NagZ in AmpC derepression, nag $Z$ gene was deleted in both derepression strains to construct YE $\Delta \mathrm{D} 123 \Delta \mathrm{Z}$ and YE $\Delta 4 \Delta 5 \Delta 6 \Delta 7 \Delta \mathrm{Z}$. As shown in Figure 2, nag $Z$ was indispensable for $a m p C$ over expression of YE $\Delta 4 \Delta 5 \Delta 6 \Delta 7$, the $\beta$-lactamase activity of $n a g Z$ deletion strain YE $\Delta 4 \Delta 5 \Delta 6 \Delta 7 \Delta \mathrm{Z}$ was decreased significantly, closed to the wild-type strain level. In complementation assay, $\mathrm{YE} \Delta 4 \Delta 5 \Delta 6 \Delta 7 \Delta \mathrm{Z}$ (pNagZ) restored the $\beta$-lactamase activity to the level of YE $\Delta 4 \Delta 5 \Delta 6 \Delta 7$. However, NagZ was dispensable in $\mathrm{YE} \Delta \mathrm{D} 123$, the $\beta$-lactamase activity of nagZ deletion strain $\mathrm{YE} \Delta \mathrm{D} 123 \Delta \mathrm{Z}$ was nearly as high as YE $\Delta \mathrm{D} 123$ (Figure 2). These results suggested that NagZ was needed in $\triangle$ PBPsdriven AmpC derepression, but did not perform its expected function in $A m p D$ mutation strains. Antibiotic susceptibility test was also performed, as shown in Table 3, the MIC values of YE $\Delta 4 \Delta 5 \Delta 6 \Delta 7 \Delta \mathrm{Z}$ were slightly below the wild-type strain $105.5 \mathrm{R}(\mathrm{r})$, far from its parent strain YE $\Delta 4 \Delta 5 \Delta 6 \Delta 7$ for almost all tested $\beta$-lactams; but only a marginal distinction between $\mathrm{YE} \Delta \mathrm{D} 123$ and $\mathrm{YE} \Delta \mathrm{D} 123 \Delta \mathrm{Z}$ was found. These results illustrated that AmpD/PBPs regulate AmpC expression through NagZ dispensable/indispensable ways in Y. enterocolitica.

\section{N-Acetyl- $\beta$-Glucosaminidase Activity Assay}

The nagZ mutation strain $\mathrm{YE} \Delta \mathrm{Z}$ was constructed, and determined by the enzyme activity of the both wild-type strain and $\mathrm{YE} \Delta \mathrm{Z}$ for $10 \mathrm{~h}$ using $\mathrm{N}$-acetyl- $\beta$-D-glucosaminide as substrate. As shown in Figure 3, YE $\Delta \mathrm{Z}$ abolished the $\mathrm{N}$-acetyl- $\beta$ glucosaminidase activity completely, it was suggested that NagZ is the only enzyme that with $\mathrm{N}$-acetyl- $\beta$-glucosaminidase activity in Y. enterocolitica.

\section{Role of AmpR in ampC Expression of in $Y$. enterocolitica}

In the paradigm of the $a m p R-a m p C$ system, the $a m p R$ gene is located immediately adjacent to $\operatorname{ampC}$, and $A m p R$ plays a pivotal role in the regulation of AmpC (Seoane et al., 1992). To assess the role of AmpR in Y. enterocolitica, we compared the $\beta$-lactamase activity of $\mathrm{YE} \Delta \mathrm{D} 123 \Delta \mathrm{R}, \mathrm{YE} \Delta 4 \Delta 5 \Delta 6 \Delta 7 \Delta \mathrm{R}$ with their parent strains $\mathrm{YE} \Delta \mathrm{D} 123, \mathrm{YE} \Delta 4 \Delta 5 \Delta 6 \Delta 7$, respectively. As a result, $a m p R$ inactivation dramatically reduced the $\beta$-lactamase 


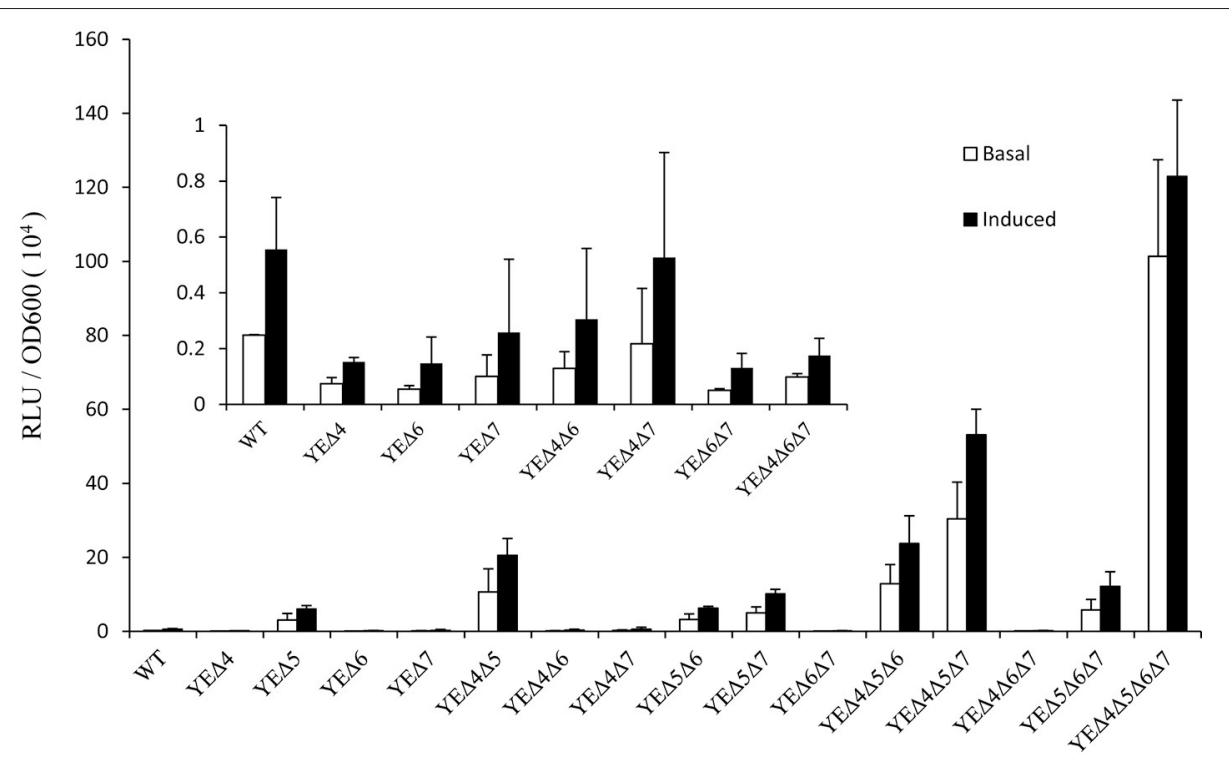

FIGURE 1 | Analysis of the ampC promoter activities in $Y$. enterocolitica 105.5R(r) wild-type strain and pbp mutants. The induction group was incubated with $40 \mu \mathrm{g} / \mathrm{ml}$ cefoxitin for $1 \mathrm{~h}$. The error bars represent the standard deviations of triplicate tests.

TABLE 2 | Relative mRNA level of ampC in wild-type strain and its derived mutants.

\begin{tabular}{lcc}
\hline Strain & \multicolumn{2}{c}{ Relative mRNA level of amp $\mathbf{c}^{\mathbf{a}}$} \\
\cline { 2 - 3 } & Basal & Induced $^{\mathbf{b}}$ \\
\hline $\mathrm{WT}$ & 1 & $1.3 \pm 0.4$ \\
YE $\Delta 4$ & $1 \pm 0.6$ & $1.7 \pm 0.5$ \\
YE $\Delta 5$ & $5.8 \pm 3.5$ & $7.8 \pm 3.0$ \\
YE $\Delta 6$ & $1.2 \pm 0.6$ & $1.8 \pm 0.6$ \\
YE $\Delta 7$ & $0.7 \pm 0.4$ & $1.2 \pm 0.5$ \\
YE $\Delta 4 \Delta 5$ & $10 \pm 5$ & $31 \pm 16$ \\
YE $\Delta 4 \Delta 6$ & $1 \pm 0.2$ & $1.4 \pm 0.4$ \\
YE $\Delta 4 \Delta 7$ & $0.7 \pm 0.2$ & $1.2 \pm 0.5$ \\
YE $\Delta 5 \Delta 6$ & $11 \pm 1$ & $15 \pm 8$ \\
YE $\Delta 5 \Delta 7$ & $7.7 \pm 1.0$ & $12 \pm 4.8$ \\
YE $\Delta 6 \Delta 7$ & $1.6 \pm 0.3$ & $2.4 \pm 1.4$ \\
YE $\Delta 4 \Delta 5 \Delta 6$ & $22 \pm 5$ & $32 \pm 18$ \\
YE $\Delta 4 \Delta 5 \Delta 7$ & $26 \pm 4$ & $41 \pm 13$ \\
YE $\Delta 4 \Delta 6 \Delta 7$ & $2.1 \pm 0.5$ & $3.3 \pm 1.6$ \\
YE $\Delta 5 \Delta 6 \Delta 7$ & $8.5 \pm 1.0$ & $12 \pm 5$ \\
YE $\Delta 4 \Delta 5 \Delta 6 \Delta 7$ & $42 \pm 23$ & $58 \pm 10$ \\
\hline R & & \\
\hline
\end{tabular}

${ }^{a}$ Relative amount of mRNA compared to wild-type strain 105.5R(r) basal expression.

${ }^{b}$ Induction assay were performance with $40 \mu \mathrm{g} / \mathrm{ml}$ cefoxitin.

activity of both YE $\Delta \mathrm{D} 123 \Delta \mathrm{R}$ and $\mathrm{YE} \Delta 4 \Delta 5 \Delta 6 \Delta 7 \Delta \mathrm{R}$, regardless of adding cefoxitin or not (Figure 2).

\section{DISCUSSION}

The ampR-ampC system from Citrobacter freundii and Enterobacter cloacae has been well studied in the early 1990s (Lindberg et al., 1987; Peter et al., 1988). However, newly discovered $a m p C$ regulators such as, PBP4 (DacB) or NagZ in Enterobacteriaceae was not yet understood. A deep study in $Y$. enterocolitica ampR-ampC system would be helpful to improve the comprehensive understanding of Enterobacteriaceae ampC regulation.

PBPs are a group of enzymes involved in cell-wall recycling and the processes of AmpC $\beta$-lactamases regulation. In E. coli model, deletion of three or four PBPs and the concomitant inhibition of PBP 1a, 1b, and/or 2 results in an increased level of $\beta$-lactamase induction (Pfeifle et al., 2000). However, since E. coli lacks the chromosomal $a m p R$ gene, the result may be inconsistent with other members of the Gram-negative bacteria which have a chromosome encoding the $\operatorname{ampR-ampC}$ system. In 2009, Moya et al. demonstrated the inactivation of DacB (PBP4), a nonessential low-molecular mass PBPs is the principal reason for one-step high-level ampC expression in clinical strains of $P$. aeruginosa (Moya et al., 2009). Interestingly, inactivation of PBP4 in E. cloacae triggered a significant increase of $\beta$ lactams resistance, but without an obvious upregulation of $\operatorname{ampC}$ gene, it may be suggested that PBP4 regulates AmpC at a posttranscriptional level (Guerin et al., 2015). In this study, we found deletion of $p b p 4$ did not elevate the $a m p C$ expression level, this result is accordance with E. cloacae. After that, we deleted all four LMM PBPs one after another, and found that PBP5 is the most effective PBP involved in the regulation of $a m p C$ in $Y$. enterocolitica. Of the single-mutation strains, only the $p b p 5$ deletion strain YE $\Delta 5$ showed an obvious rise in ampC expression level. Likewise, for multi-mutation strains, the function of PBP4, PBP6, and PBP7 in ampC regulation were detected only if in $\Delta p b p 5$ background. According to the results shown in Figure 1 and Table 2, we deduced the hierarchy of the role of PBPs genes in ampC derepression: PBP5 > PBP4 > PBP7 > PBP6. Although $\mathrm{DacB}$ may regulates $\mathrm{AmpC}$ at a post-transcriptional level (Guerin 


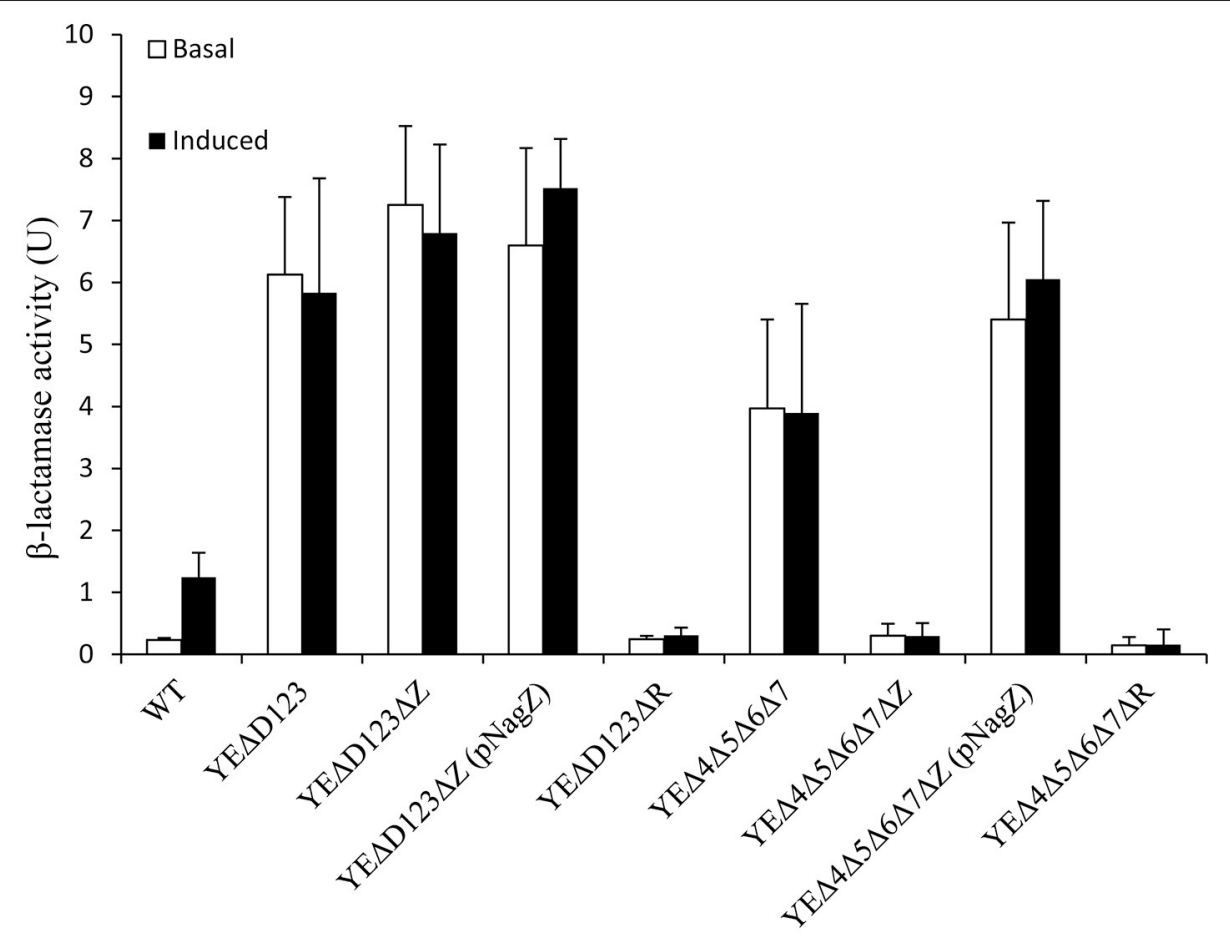

FIGURE 2 | The role of AmpD, PBPs, NagZ, and AmpR in the $\beta$-lactamase expression of $Y$. enterocolitica by measuring the $\beta$-lactamase activity. These data are the average of three repeat experiments. The induction group was incubated with $40 \mu \mathrm{g} / \mathrm{ml}$ cefoxitin for $1 \mathrm{~h}$. Error bars indicate the standard deviations of triplicate tests.

TABLE 3 | The MIC values of $\beta$-lactam antibiotics in wild-type strain and its derived mutants.

\begin{tabular}{|c|c|c|c|c|c|}
\hline Antibiotic & \multicolumn{5}{|c|}{ MIC (mg/L) of antibiotic of strain ${ }^{a, b}$} \\
\hline AMP & 32 & 64 & 32 & 64 & 16 \\
\hline SAM & 16 & 16 & 16 & 16 & 8 \\
\hline TIC & 2 & 4 & 2 & 4 & 0.5 \\
\hline \multicolumn{6}{|c|}{ CEPHALOSPORINS } \\
\hline CFZ & 128 & 512 & 512 & 512 & 64 \\
\hline CAZ & 0.25 & 2 & 1 & 2 & 0.5 \\
\hline FEP & 0.25 & 0.25 & 0.125 & 0.06 & 0.03 \\
\hline CRO & $\leq 0.125$ & 0.5 & 0.25 & 0.5 & 0.125 \\
\hline IPM & $\leq 0.125$ & 0.5 & 0.25 & 0.25 & 0.25 \\
\hline MEM & $\leq 0.125$ & $\leq 0.125$ & $\leq 0.125$ & $\leq 0.125$ & $\leq 0.125$ \\
\hline \multicolumn{6}{|c|}{ LIPOPEPTIDES } \\
\hline CL & $\leq 0.5$ & $\leq 0.5$ & $\leq 0.5$ & 0.75 & $\leq 0.5$ \\
\hline
\end{tabular}

a AMP, Ampicillin; SAM, Ampicillin-sulbactam; TIC, Ticarcillin; TZP, Piperacillin-tazobactam; PIP, Piperacillin; CFZ, Cefazolin; CAZ, Ceftazidime; FEP, Cefepime; CRO, Ceftriaxone; ATM, Aztreonam; IPM, Imipenem; MEM, Meropenem; CL, Colistin.

${ }^{b}$ MIC was determined in triplicate by standard two-fold serial broth microdilution method. 


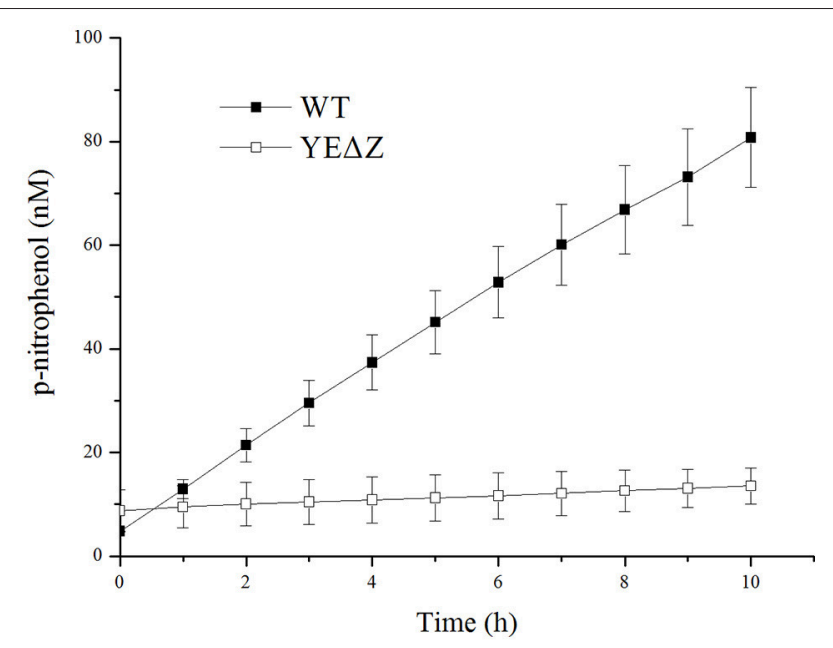

FIGURE 3 | The $\mathrm{N}$-acetyl- $\beta$-glucosamididase activity of wild-type $Y$. enterocolitica and nag $Z$ deletion mutants was tested using 4-nitrophenyl $\mathrm{N}$-acetyl- $\beta$-D-glucosaminide as a chromogenic substrate, and the $\mathrm{p}$-nitrophenol present in the supernatant was measured at $405 \mathrm{~nm}$.

et al., 2015), but no trace of post-transcriptional mechanism has been found in Y. enterocolitica.

Along with the popular research of $\operatorname{ampC}$ regulation, there is growing evidence that some bacteria may regulate the expression of $a m p C$ through at least two different ways, one of which was NagZ-dependent, while the other worked without the participation of NagZ (Huang et al., 2012; Guerin et al., 2015). In the study on $P$. aeruginosa, nag $Z$ inactivation was shown to attenuate $\operatorname{ampC}$ expression and was critical for basallevel $a m p C$ derepression in both $\mathrm{PA} \triangle \mathrm{D}$ ( $a m p D$ inactivation) and PA $\Delta \mathrm{dB}$ ( $p b p 4$ inactivation) mutants (Asgarali et al., 2009; Zamorano et al., 2010). However, $\Delta$ nagZ had little effect on the cefoxitin-induced ampC expression level in both PA $\Delta \mathrm{D}$ and $\mathrm{PA} \Delta \mathrm{dB}$, which indicated that an unidentified non-NagZ product at work in this induction process. Furthermore, two different regulation ways of $\beta$-lactamase have been found in S. maltophilia, on one hand NagZ was essential for $\mathrm{KJ} \Delta \mathrm{DI}$ ( $a m p D$ inactivation) $a m p C$ overexpression, on the other hand, $n a g Z$ inactivation hardly influenced the ampC expression level of KJ $\Delta$ mrcA (pbp1a inactivation; Huang et al., 2012). In this study, we also found two different $\operatorname{ampC}$ regulation ways exist in $Y$. enterocolitica, the patterns of which were just the reverse of that in S. maltophilia (Huang et al., 2012). The $\beta$-lactamase activity of YE $\triangle \mathrm{D} 123$ was not affected by the inactivation of the nag $Z$ gene, whereas the introduction of $\triangle$ nag $Z$ into the PBP mutation strain YE $\Delta 4 \Delta 5 \Delta 6 \Delta 7$ dramatically reduced the $\beta$-lactamase activities at both the basal and induced level (Figure 2). As shown in Table 3, the antibiotic resistance of YE $\Delta 4 \Delta 5 \Delta 6 \Delta 7$ and YE $\Delta$ D 123 were marked improved compare with wild-type strain, the MIC value of these two strains in TZP, PIP, CFZ, CAZ, CRO, and ATM is rising sharply. While after inactivation of nagZ gene simultaneously, only a marginal distinction between $\mathrm{YE} \Delta \mathrm{D} 123$ and $\mathrm{YE} \Delta \mathrm{D} 123 \Delta \mathrm{Z}$ was found, but the MIC values of YE $\Delta 4 \Delta 5 \Delta 6 \Delta 7 \Delta \mathrm{Z}$ has shifted down significantly, far from its parent strain YE $\Delta 4 \Delta 5 \Delta 6 \Delta 7$ for almost all tested $\beta$-lactams. To further confirm the function of NagZ, we constructed a nagZ deletion strain $\mathrm{YE} \Delta \mathrm{Z}$, and detected the $\mathrm{N}$-acetyl- $\beta$-glucosaminidase activity of it to compare with the wild-type strain Y. enterocolitica 105.5R(r), the results showed that the ability of hydrolysis chromogenic substrate was completely lost in nagZ mutation strain $\mathrm{YE} \Delta \mathrm{Z}$ (Figure 3), suggesting that NagZ (YE105_RS06670) was the only enzyme that possessed $\mathrm{N}$-acetyl- $\beta$-glucosaminidase activity in $Y$. enterocolitica $105.5 \mathrm{R}(\mathrm{r})$. However, even though there is no readable $\mathrm{N}$-acetyl- $\beta$-glucosaminidase activity in $\mathrm{YE} \Delta \mathrm{Z}$, we also did the bioinformatic search to look for possible NagZ homologs in genome to find the protein worked in YE $\Delta \mathrm{D} 123 \Delta \mathrm{Z}$. According to the gene function annotation of $105.5 \mathrm{R}(\mathrm{r})$, we considered the YE105_RS13000 may have similar function with $\mathrm{NagZ}$, but it was not clear if this protein participated the ampC regulation or not. Therefore, further studies needed to performed to elucidate the function of YE105_RS13000 in Y. enterocolitica ampC regulation.

In $Y$. enterocolitica, the function of AmpR was roughly the same as other members of Enterobacteriaceae or P. aeruginosa. The introduction of $\triangle a m p R$ into the AmpC hyperproduction strains $\mathrm{YE} \Delta \mathrm{D} 123$ and $\mathrm{YE} \Delta 4 \Delta 5 \Delta 6 \Delta 7$ resulted in a sharp decline in the ampC expression (Figure 2). The inducibility of $\mathrm{YE} \Delta \mathrm{D} 123 \Delta \mathrm{R}$ and $\mathrm{YE} \Delta 4 \Delta 5 \Delta 6 \Delta 7 \Delta \mathrm{R}$ also disappeared completely (Lindberg et al., 1985; Lindberg and Normark, 1987).

In conclusion, in terms of AmpC $\beta$-lactamase regulation, $Y$. enterocolitica shared some common characteristics with $P$. aerugiosa and other members of Enterobacteriaceae, but it also had its own features. This was the first investigation to the characterization of $Y$. enterocolitica ampC regulation. It provided a more comprehensive understanding of the AmpC $\beta$-lactamase regulation in Gram-negative bacteria.

\section{AUTHOR CONTRIBUTIONS}

CL, CCL, SS, HJ, and XW designed the experiment together. YC and $\mathrm{HH}$ performed data analysis. JL and $\mathrm{RD}$ participated in the manuscript translation. ZG, JZ, and ZZ contributed to finish the work. All authors contributed to writing of the manuscript.

\section{FUNDING}

This work was supported by the National Natural Science Foundation of China (General Project, no. 81470092) and the National Sci-Tech Key Project (2012ZX10004201, 2013ZX10004203-002).

\section{ACKNOWLEDGMENTS}

We thank Liuying Tang and American Journal Experts for their critical reading and helpful comments on our manuscript (Sub ID F3Y8N2N7). 


\section{REFERENCES}

Asgarali, A., Stubbs, K. A., Oliver, A., Vocadlo, D. J., and Mark, B. L. (2009). Inactivation of the glycoside hydrolase NagZ attenuates antipseudomonal $\beta$ lactam resistance in Pseudomonas aeruginosa. Antimicrob. Agents Chemother. 53, 2274-2282. doi: 10.1128/AAC.01617-08

Balasubramanian, D., Kumari, H., and Mathee, K. (2015). Pseudomonas aeruginosa AmpR: an acute-chronic switch regulator. Pathog. Dis. 73, 1-14. doi: 10.1111/2049-632X.12208

Bent, Z. W., and Young, G. M. (2010). Contribution of BlaA and BlaB $\beta$-lactamases to antibiotic susceptibility of Yersinia enterocolitica biovar 1B. Antimicrob. Agents Chemother. 54, 4000-4002. doi: 10.1128/AAC.01754-09

Chen, Y., Duan, R., Li, X., Li, K., Liang, J., Liu, C., et al. (2015). Homology analysis and cross-immunogenicity of OmpA from pathogenic Yersinia enterocolitica, Yersinia pseudotuberculosis, and Yersinia pestis. Mol. Immunol. 68(2 Pt A), 290-299. doi: 10.1016/j.molimm.2015.09.016

CLSI (2015). Performance Standards for Antimicrobial Susceptibility Testing; Twenty-Fourth Informational Supplement (M100-S24). Wayne, PA.

Cornelis, G., and Abraham, E. P. (1975). Beta-lactamases from Yersinia enterocolitica. J. Gen. Microbiol. 87, 273-284. doi: 10.1099/00221287-87-2-273

Guerin, F., Isnard, C., Cattoir, V., and Giard, J. C. (2015). Complex regulation pathways of AmpC-mediated $\beta$-lactam resistance in Enterobacter cloacae complex. Antimicrob. Agents Chemother. 59, 7753-7761. doi: 10.1128/AAC.01729-15

Huang, Y. W., Hu, R. M., Lin, C. W., Chung, T. C., and Yang, T. C. (2012). NagZdependent and NagZ-independent mechanisms for $\beta$-lactamase expression in Stenotrophomonas maltophilia. Antimicrob. Agents Chemother. 56, 1936-1941. doi: 10.1128/AAC.05645-11

Juan, C., Moya, B., Perez, J. L., and Oliver, A. (2006). Stepwise upregulation of the Pseudomonas aeruginosa chromosomal cephalosporinase conferring highlevel $\beta$-lactam resistance involves three AmpD homologues. Antimicrob. Agents Chemother. 50, 1780-1787. doi: 10.1128/AAC.50.5.1780-1787.2006

Liang, J., Li, X., Zha, T., Chen, Y., Hao, H., Liu, C., et al. (2016). DTDP-rhamnosyl transferase $\mathrm{RfbF}$, is a newfound receptor-related regulatory protein for phage phiYe-F10 specific for Yersinia enterocolitica serotype O:3. Sci. Rep. 6:22905. doi: $10.1038 /$ srep 22905

Liang, J., Wang, X., Xiao, Y., Cui, Z., Xia, S., Hao, Q., et al. (2012). Prevalence of Yersinia enterocolitica in pigs slaughtered in Chinese abattoirs. Appl. Environ. Microbiol. 78, 2949-2956. doi: 10.1128/AEM.07893-11

Lindberg, F., and Normark, S. (1987). Common mechanism of ampC beta-lactamase induction in enterobacteria: regulation of the cloned Enterobacter cloacae P99 beta-lactamase gene. J. Bacteriol. 169, 758-763. doi: $10.1128 /$ jb.169.2.758-763.1987

Lindberg, F., Lindquist, S., and Normark, S. (1987). Inactivation of the ampD gene causes semiconstitutive overproduction of the inducible Citrobacter freundii $\beta$-lactamase. J. Bacteriol. 169, 1923-1928. doi: 10.1128/jb.169.5.1923-1928. 1987

Lindberg, F., Westman, L., and Normark, S. (1985). Regulatory components in Citrobacter freundii ampC beta-lactamase induction. Proc. Natl. Acad. Sci. U.S.A. 82, 4620-4624. doi: 10.1073/pnas.82.14.4620

Liu, C., Wang, X., Chen, Y., Hao, H., Li, X., Liang, J., et al. (2016). Three Yersinia enterocolitica AmpD homologs participate in the multi-step regulation of chromosomal Cephalosporinase, AmpC. Front. Microbiol. 7:1282. doi: $10.3389 /$ fmicb.2016.01282

Moya, B., Dotsch, A., Juan, C., Blazquez, J., Zamorano, L., Haussler, S., et al. (2009). $\beta$-lactam resistance response triggered by inactivation of a nonessential penicillin-binding protein. PLoS Pathog. 5:e1000353. doi: 10.1371/journal.ppat.1000353

Peter, K., Korfmann, G., and Wiedemann, B. (1988). Impact of the ampD gene and its product on beta-lactamase production in Enterobacter cloacae. Rev. Infect. Dis. 10, 800-805. doi: 10.1093/clinids/10.4.800

Pfeifle, D., Janas, E., and Wiedemann, B. (2000). Role of penicillinbinding proteins in the initiation of the AmpC $\beta$-lactamase expression in Enterobacter cloacae. Antimicrob. Agents Chemother. 44, 169-172. doi: 10.1128/AAC.44.1.169-172.2000

Philippe, N., Alcaraz, J. P., Coursange, E., Geiselmann, J., and Schneider, D. (2004). Improvement of pCVD442, a suicide plasmid for gene allele exchange in bacteria. Plasmid 51, 246-255. doi: 10.1016/j.plasmid.2004.02.003

Ropy, A., Cabot, G., Sanchez-Diener, I., Aguilera, C., Moya, B., Ayala, J. A., et al. (2015). Role of Pseudomonas aeruginosa low-molecular-mass penicillin-binding proteins in AmpC expression, $\beta$-lactam resistance, and peptidoglycan structure. Antimicrob. Agents Chemother. 59, 3925-3934. doi: 10.1128/AAC.05150-14

Sanders, C. C., Bradford, P. A., Ehrhardt, A. F., Bush, K., Young, K. D., Henderson, T. A., et al. (1997). Penicillin-binding proteins and induction of AmpC $\beta$ lactamase. Antimicrob. Agents Chemother. 41, 2013-2015.

Seoane, A., Francia, M. V., and Garcia Lobo, J. M. (1992). Nucleotide sequence of the ampC-ampR region from the chromosome of Yersinia enterocolitica. Antimicrob. Agents Chemother. 36, 1049-1052. doi: 10.1128/AAC.36. 5.1049

Simon, R., Priefer, U., and Puhler, A. (1983). A broad host range mobilization system for in vivo genetic engineering: transposon mutagenesis in gram negative bacteria. Nat. Biotechnol. 1, 784-791. doi: 10.1038/nbt1183-784

Vollmer, W., Joris, B., Charlier, P., and Foster, S. (2008). Bacterial peptidoglycan (murein) hydrolases. FEMS Microbiol. Rev. 32, 259-286. doi: 10.1111/j.1574-6976.2007.00099.x

Wang, X., Li, Y., Jing, H., Ren, Y., Zhou, Z., Wang, S., et al. (2011). Complete genome sequence of a Yersinia enterocolitica "Old World" (3/O:9) strain and comparison with the "New World" (1B/O:8) strain. J. Clin. Microbiol. 49, 1251-1259. doi: 10.1128/JCM.01921-10

Yang, T. C., Chen, T. F., Tsai, J. J., and Hu, R. M. (2014). NagZ is required for beta-lactamase expression and full pathogenicity in Xanthomonas campestris pv. campestris str. 17. Res. Microbiol. 165, 612-619. doi: 10.1016/j.resmic.2014.08.008

Zamorano, L., Reeve, T. M., Deng, L., Juan, C., Moya, B., Cabot, G., et al. (2010). NagZ inactivation prevents and reverts $\beta$-lactam resistance, driven by AmpD and PBP 4 mutations, in Pseudomonas aeruginosa. Antimicrob. Agents Chemother. 54, 3557-3563. doi: 10.1128/AAC.00385-10

Zeng, X., and Lin, J. (2013). Beta-lactamase induction and cell wall metabolism in Gram-negative bacteria. Front. Microbiol. 4:128. doi: 10.3389/fmicb.2013.00128

Conflict of Interest Statement: The authors declare that the research was conducted in the absence of any commercial or financial relationships that could be construed as a potential conflict of interest.

Copyright (c) 2017 Liu, Li, Chen, Hao, Liang, Duan, Guo, Zhang, Zhao, Jing, Wang and Shao. This is an open-access article distributed under the terms of the Creative Commons Attribution License (CC BY). The use, distribution or reproduction in other forums is permitted, provided the original author(s) or licensor are credited and that the original publication in this journal is cited, in accordance with accepted academic practice. No use, distribution or reproduction is permitted which does not comply with these terms. 